\title{
Predicting STOP-Sign Compliance at All-Way Stop Intersections in Close Proximity to Signalized Intersections
}

\author{
Stephen A. Arhin \\ Ph.D., P.E., PTOE, PMP ${ }^{a^{*}}$ \\ ${ }^{a}$ Howard University Transportation Research Center \\ 2300 Sixth Street, NW, Washington, DC 20059 \\ Adam Gatiba ${ }^{b}$ \\ ${ }^{\mathrm{b}}$ Howard University Transportation Research Center \\ 2300 Sixth Street, NW, Washington, \\ DC 20059
}

\begin{abstract}
STOP-signs (at unsignalized intersections) that are in close proximity to signalized intersections are often violated by drivers while "speeding up" to go through the upstream or downstream signalized intersection that have the green interval upon approach. It is thought that if the distance between the upstream or downstream signalized and the AWSC intersection is long, drivers usually comply with the STOP signs at the AWSC intersection. This research determined driver compliance rates (CRs) at All-Way STOP Control (AWSC) intersections that are in close proximity to upstream or downstream signalized intersections and, explored the existence of a relationship between $C R$ and the distance between a pair of signalized and AWSC intersections. Thirty (30) isolated segments with combinations of signalized and AWSC intersections in the District of Columbia were selected for the study. Field data (traffic volumes) were obtained at each intersection in addition to observation of driver compliance with STOP signs at AWSC intersections via video playback. In all, 13,956 observations were made at 57 AWSC intersections in 2017. The study showed that lower CRs were observed at AWSC intersections that are in closer proximity to the signalized intersections. Thus, the shorter the distance from the existing AWSC to signalized intersections, the lower the CR (or higher violation rate). Based on the data obtained, a non-linear relationship between $C R$ and distance between pairs of intersections was developed. From the model, to achieve a minimum compliance rate of $95 \%$ at an AWSC, an optimal distance of approximately 1,300 feet between the intersections is required.
\end{abstract}

Keywords: Compliance Rate, All-Way STOP Controls, Signalization

\section{INTRODUCTION}

Most intersections in urban areas are either signalized or unsignalized (STOP or YIELD-Controlled). As a result road segments in such areas have a combination of both types of intersections. In order to ensure safety of drivers and pedestrians, it important for drivers to comply with traffic control devices. While compliance with traffic signals is relatively high, compliance with STOP signs is relatively low, especially for unsignalized intersections that are close to those signalized. Drivers often violate STOP signs in order to travel through the next signalized intersection that may have the green interval on approach.

\author{
Melissa Anderson ${ }^{c}$ \\ ${ }^{\mathrm{c}}$ Howard University Transportation Research Center \\ 2300 Sixth Street, NW, Washington, \\ DC 20059 \\ Melkamsew Ribbisso ${ }^{\mathrm{d}}$ \\ ${ }^{\mathrm{d}}$ Howard University Transportation Research Center \\ 2300 Sixth Street, NW, Washington, \\ DC 20059
}

This research explores the existence of a relationship between STOP sign compliance rate and the distance between an AWSC and a signalized intersection (downstream or upstream). Thirty segments with combinations of signalized and AWSC intersections in an urban area (Washington DC) were selected for this study. Each segment consisted of at least two signalized and one AWSC intersections. STOP sign compliance was observed via video playback during the off-peak period (10:00AM to 1:00PM) based on which the compliance rate (CR) at each AWSC intersection was then computed. A model was then developed to predict $\mathrm{CR}$ based on the distance between AWSC and upstream or downstream signalized intersections.

\section{LITERATURE REVIEW}

Traffic flow interruptions are most likely to originate at or near intersections. Ideally, it is recommended that the minimum spacing for intersections in urban areas is 0.5 miles [1]. However, in some urban areas, intersections are much more closely spaced since there are often competing needs for providing land access. When access to an intersection is regulated by traffic signals or regulatory signs, it said to be controlled, while it is uncontrolled when access is regulated by the right-of-way rules. Controlled intersections are either signalized or unsignalized. Signalized intersections are controlled by traffic lights while unsignalized intersections are controlled by either STOP signs or yield signs. The STOP signs are installed either on the minor-roads only (Two-Way STOP control-TWSC) or on all approaches (All-Way STOP control-AWSC).

Roadway networks in dense urban areas consist of combinations of signalized and unsignalized intersections which may affect throughput and may have safety implications. The Federal Highway Administration (FHWA) reported that since 2005, there has been a decline in the overall number of crashes that occur at or in close proximity to intersections [2]. Nevertheless, crashes at intersections are still a major concern for traffic authorities. In 2015 , a total of 48,923 vehicles were involved in fatal crashes in the United States. Out of the total, approximately $4.4 \%(2,157)$ of the fatal crashes occurred at STOP- 
controlled intersections, while $7.5 \%(3,672)$ of the crashes occurred at intersections controlled by traffic signals. On the other hand, intersections without any type of traffic control device, recorded the highest number of fatal crashes $(4,227)$ [3]. Most of these crashes occur due to the failure of a driver to stop or yield the right-of-way.

\subsection{Strategies for Improving Mobility on Corridors}

Several studies have been conducted to determine factors that affect drivers' compliance to the right-of-way rules at intersections. In 1977, Mounce concluded that traffic volumes on a major-roadway has a strong negative relationship with the total violation rate. According to Mounce, as traffic volume increases, the total number of violations decreased. This was established by observing motorists' compliance at 66 unsignalized intersections in south central Texas, during which a total of 2,830 vehicles were observed. The variables considered in this study were major-roadway volume, minor roadway sight distance, traffic conditions and intersection geometry [4].

Mounce observed three levels of compliance: full compliance, partial violation and complete violation. A vehicle was considered to be fully compliant if it came to a complete stop at the STOP sign. A partial violation was defined as when a vehicle entered the intersection with a rolling stop at a speed of approximately 5 miles per hour (mph). A complete violation is when a vehicle does not stop before entering the intersection with a speed of $5 \mathrm{mph}$ or more. The compliance rate of each intersection was then calculated. A factorial experimental design was utilized to determine which variable influenced the compliance rate the most. The results revealed that major roadway volume significantly affected compliance rate. Also, the interaction effect of major roadway volumes and minor road sight distance significantly affected compliance rate. Intersection geometry type was determined not to contribute to the rate of compliance. From the results, Mounce recommended that STOP signs should be installed on minor streets where major street volumes are low in order to improve throughput [4]. In 2012, Woldeamanuel conducted an observational study to examine how drivers' behavior at STOP-controlled intersections is affected by different socio-demographic and physical attributes. The variables used in this study included gender, age, number of passengers, time of day, urban setting, presence of law enforcement and headlight usage. A total of 2,400 observations were made at four (4) major intersections in Saint Cloud, Minnesota. The stop events were classified as complete, rolling or no stop. The results of the study revealed that $35 \%$ of the drivers made a complete stop, whereas $65 \%$ of them did not make a complete stop (52\% made a rolling stop and $13 \%$ did not make any stop at all). The variables age, number of passengers in the vehicle, time of day, presence of law enforcement and headlight usage were determined to significantly influence the behavior of drivers. The results indicate that older drivers were more likely to comply with STOP signs at intersections. Also, the likelihood of making a complete stop increased with the number of passengers in the vehicle and, it was more likely that drivers would come to a complete stop at night than during the day. From the results, presence of police enforcement increased the likelihood of drivers coming to a complete stop. Finally, the likelihood of drivers making a complete stop decreased with less headlight usage [5].

In 2017, Mahadiow investigated drivers' compliance at AWSC intersections in close proximity to signalized intersections. Drivers' compliance was observed at eight AWSC intersections that were at most 600 feet away to an upstream/downstream signalized intersection. Higher compliance rates were observed for vehicles which were heading towards the signalized intersections than vehicles moving away from the signalized intersections. The study further established a linear relationship between drivers' compliance and distance between the intersections [6].

\subsection{Factors influencing non-compliance at signalized intersections}

Non-compliance is not limited to only unsignalized intersections. In 1986, Gordon and Robertson studied the cause of driver noncompliance at twelve (12) signalized intersections in the Washington, D.C., metropolitan area. The study further sought to define a relationship between noncompliance, intersection operational characteristics (traffic volumes) and roadway features such as number of approach lanes, traffic signal location, intersection jurisdiction and primary land use. Violations were observed for vehicles making left, through and right turn movements at the intersections. About 380 observations were made at each intersection. The results showed that higher violations occurred at intersections with low traffic volumes. Also, most violations occurred during the off-peak periods. The highest number of violations was recorded for vehicles making right turn at intersections where right turn on red is permitted. Violating drivers usually failed to come to a complete stop before turning right. Fewer vehicles were observed making through and left turn movements on red signal. Overall, an average of 1.25 violations occurred for every 100 vehicles entering an intersection [7].

In 2010, Elmitiny investigated how certain variables affect a driver's decision to stop or go on a red-light, at signalized intersections. The variables used included distance of the vehicle from the STOP bar, approach speed, yellow interval entry time, whether the vehicle was in the lead or following position, lane position, and vehicle type. The study intersection was located on a high-speed corridor located in a suburb in Central Florida. Data was collected using a threecamera-video-based system which recorded drivers' behavior associated with traffic signal change. A total of 1,292 vehicle observations were made. The results of the statistical analysis showed that the speeds of vehicles significantly affected drivers' decision to stop or go. Vehicles which approached the intersection at higher speeds were more likely to violate the red light. Also, the probability of a stop decision increased as the distance of the vehicle from the STOP line increased. Drivers within 280 feet to 320 feet from the intersection had probabilities of both stop and go decisions close to $50 \%$. This implies that vehicles within this interval showed the largest variability in their decision to stop or go during the yellow interval. Also, $90 \%$ of red-light-violators were within the 210 feet to 480 feet range of the intersection [8]. 
In 2013, Chuanyun et al also examined the contributing factors affecting compliance of traffic signs and signals. The study concluded that at signalized intersections, incoming drivers are usually puzzled whether to speed up or slow down when the traffic signal changes from green to yellow. If they are far from the traffic signal, they tend to speed through the intersection and this may lead to a traffic signal violation. If they suddenly slow down, it may result in rearend collision [9].

\subsection{Countermeasures for Traffic Control Signals and STOP Sign Violations}

Various measures have been tested to mitigate the violation of traffic signals and STOP signs. In 2009, Rice and Polanis showcased low-cost plans to improve safety at four STOPcontrolled intersections in Winston-Salem, North Carolina. Various measures were taken to improve visibility and pavement markings at the intersections. These included the replacement of existing 24-inch STOP signs with 30-inch STOP signs, installing "STOP AHEAD" signs before the STOP signs, and providing double yellow centerlines and stop bar pavement markings. This treatment reduced crashes by $56.7 \%$ and improved throughput [10].

Recently, advanced techniques including the use of infrastructure and vehicle-based collision avoidance systems are being implemented to increase compliance, thereby improving safety. These systems utilize roadway sensors, processors, warning devices, roadside-vehicle communication devices and other informational and warning devices to provide driving assistance to road users. Currently, connected vehicle-based approaches are being proposed to improve safety at intersections. These technologies enable the real-time sharing of vehicle data such as position, speed and acceleration [11].

The body of literature suggests that the factors which affect violations of STOP signs mainly include traffic volumes on the major roadway and socio-demographic and physical attributes such as gender, age, number of passengers, as well as the presence of law-enforcement. Also, though the literature shows that distance between CR at an AWSC intersection varies based on the distance to an adjacent signalized intersection, the existence of a non-linear relationship between CR and the distance between a pair of signalized and AWSC intersections is yet to be explored. This research therefore seeks to examine the existence of such a relationship by analyzing data collected over a wider range of locations than previous research.

\section{RESEARCH METHODOLOGY}

\subsection{Selection of Study Segments}

A total of thirty (30) segments located on arterial and collector roads were selected in the District of Columbia for this study. Each segment consists of at least two signalized intersections and one AWSC intersection. The segments are such that no two signalized intersections are successive with at most two AWSC intersections in between. A typical segment configuration is presented in Figure 1.

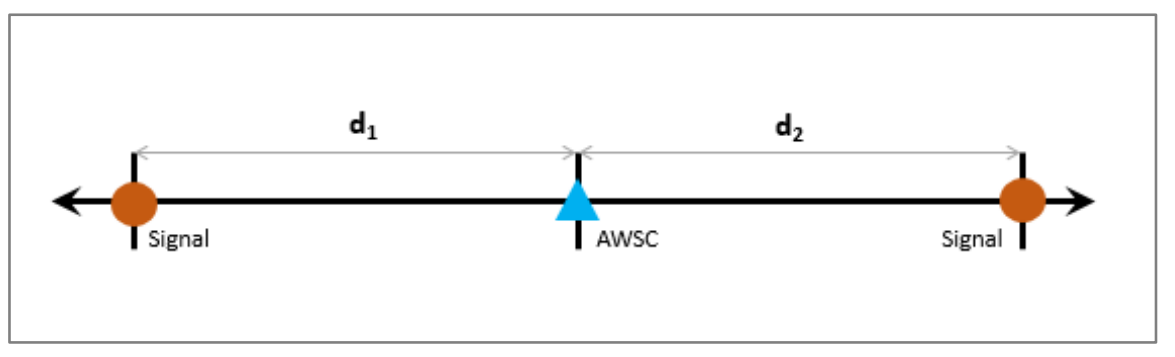

Figure 2 Study Segment Configuration

\subsection{Data Collection}

Field data collection was conducted at the thirty (30) selected segments on typical weekdays (Tuesday, Wednesday and Thursday) from April 2017 through December 2017. In the event that road maintenance or construction was ongoing at any of the intersections, the data collection was deferred until it was completed. The data collection was conducted via video recordings. Video recording cameras were installed at the STOP controlled intersections which are adjacent to the signalized intersections. The video recordings were conducted on typical weekdays (Tuesday, Wednesday and Thursday) over a twelve (12) hour duration from 6:30 AM to 6:30 PM. The following data/information associated with intersection traffic operations were obtained:

\section{a. Vehicular Volumes}

The volume of vehicles travelling upstream or downstream toward the signalized intersections during the off-peak period from 10:00AM to 1:00PM, were extracted from video playbacks. The volume counts were conducted using JAMAR count boards which were downloaded and processed.

b. STOP Sign Compliance at AWSC Intersections

From the videos, observations were made at the AWSC intersections within the segments that are in close proximity to the upstream or downstream signalized intersections. Vehicular compliance at the STOP sign was observed during the off-peak period and was classified as either compliant or non-compliant. A vehicle was in compliance if it came to a complete stop at the STOP bar for at least one (1) second before proceeding through the intersection. On the other hand, a vehicle was deemed not in compliance if it did not come to a complete stop before entering the intersection. In all, 13,956 observations were made at 57 AWSC intersections after which the data was entered into Microsoft Excel and SPSS for analysis. 


\subsection{Data Analysis}

A compliance rate (CR) analysis was conducted to obtain parameters for further statistical analyses. The CR for each of the selected AWSC intersections that were in close proximity to the upstream or downstream signalized intersections were computed using the following equation:

$$
\text { Compliance Rate }=\text { VC/TV } \times 100
$$

Where

$$
\begin{aligned}
V C= & \text { Number of vehicles in compliance, i.e., } \\
& \text { those that completely stopped before } \\
& \text { proceeding through the intersection, and }
\end{aligned}
$$

$\mathrm{TV}=$ Total number of vehicles going through the intersection on the same approach

\subsection{Statistical Analysis}

Descriptive statistics such as the mean, median, and standard deviation, were computed for $\mathrm{CR}$ and distance between successive signalized and unsignalized intersections located within the selected segments of study.

\subsection{Model Development}

To determine whether there exists a relationship between the distance between an AWSC intersection and an upstream or downstream signalized intersection, and the compliance rate (CR), a non-linear model was determined to assume the following form:

$$
C R=k_{0}+k_{1} \exp \left(k_{2} D\right)+\varepsilon
$$

where:

$C R=$ Compliance Rate $(\%)$.

$D=$ Distance between signalized and

AWSC intersections (ft)

The value $k_{0}$ is the model constant and $k_{1}$ and $k_{2}$ are the model coefficients with an associated error of $\varepsilon\left[\varepsilon \sim N\left(0, \sigma^{2}\right)\right]$. The form of the model was the result of several data transformations since the data was determined not to follow the normal distribution. A scatter plot of CR and D showed no issue with heteroscedasticity.

\section{RESULTS}

\subsection{Statistical Analysis}

The summaries of the descriptive statistical analysis for the $\mathrm{CR}$, and distance between successive signalized and unsignalized intersections located within the selected segments of study were computed and are presented in Table 1. The reported descriptive statistics are the mean, median, standard deviation and $95 \%$ confidence interval. From the table, it can be observed that the mean compliance rate was $69.84 \%$, with a standard deviation of $10.53 \%$. The highest and lowest observed compliance rates were $92.27 \%$ and $55.00 \%$ respectively. Also, the mean distance between consecutive signalized and unsignalized intersections was 461.15 feet with a standard deviation of 169.82 feet. The highest and lowest distances measured were 885 feet and 142 feet respectively.

TABLE 1 Summary of descriptive statistics for STOP sign compliance

\begin{tabular}{|c|c|c|c|c|c|c|}
\hline \multirow{2}{*}{ Variable } & \multicolumn{5}{|c|}{ Descriptive Statistics } \\
\cline { 2 - 6 } & Mean & \multirow{2}{*}{$\begin{array}{c}\text { Standard } \\
\text { Deviation }\end{array}$} & $\begin{array}{c}\text { Lowest } \\
\text { Value }\end{array}$ & $\begin{array}{c}\text { Highest } \\
\text { Value }\end{array}$ & \multicolumn{2}{c|}{ 95\% Confidence Interval } \\
\cline { 2 - 6 } & & 10.53 & 55.00 & 92.27 & 66.99 \\
\hline Compliance Rate (\%) & 69.84 & 169.82 & 142.00 & 885.00 & 415.24 \\
\hline $\begin{array}{c}\text { Distance (ft) to Signalized } \\
\text { Intersection }\end{array}$ & 461.15 & & & & 72.68 \\
\end{tabular}

\subsection{Model Development}

An analysis was conducted to determine the existence of a relationship between the distance between an AWSC intersection and signalized intersection and the CR. A model was then developed to predict the CR based on the distance. The model was assumed to take the form:

$$
C R=k_{0}+k_{1} \exp \left(k_{2} D\right)+\varepsilon
$$

The results in Table 2 show the estimates of the model coefficients. The coefficients $\mathrm{k}_{0}, \mathrm{k}_{1}$ and $\mathrm{k}_{2}$ were estimated to be $99.99,-66.90$ and -0.002 respectively. Also, the $\mathrm{R}^{2}$ value of 0.738 shown in Table 3 indicates that the model explains a high percentage $(73.8 \%)$ of the variance in the data.

TABLE 2 Regression Coefficients

\begin{tabular}{|c|c|c|c|c|}
\hline \multirow{2}{*}{ Parameter } & Estimate & \multirow{2}{*}{$\begin{array}{c}\text { Std. } \\
\text { Error }\end{array}$} & $\begin{array}{l}\text { L5\% Confidence Interval } \\
\text { Bound }\end{array}$ & Upper Bound \\
\hline $\mathrm{K}_{0}$ & 99.990 & 13.776 & 72.345 & 127.635 \\
\hline $\mathrm{K}_{1}$ & -66.900 & 8.102 & -83.158 & -50.642 \\
\hline $\mathrm{K}_{2}$ & -.002 & .001 & -.004 & $-6.161 \mathrm{E}-5$ \\
\hline
\end{tabular}

TABLE 3 ANOVA results

\begin{tabular}{|l|c|c|c|}
\hline Source & Sum of Squares & df & Mean Squares \\
\hline Regression & 272673.183 & 3 & 90891.061 \\
\hline Residual & 1565.600 & 52 & 30.108 \\
\hline Uncorrected Total & 274238.783 & 55 & \\
\hline Corrected Total & 5983.953 & 54 & \\
\hline Dependent variable: Compliance Rate \\
\hline R squared = 1 - (Residual Sum of Squares) / (Corrected Sum of Squares) $=.738$. \\
\hline
\end{tabular}


The model was therefore determined to be $C R=99.99-$ $66.907 \exp (-0.002 D)$

\subsection{Model Tests}

\section{Residual Plots}

For a statistically significant model, the residuals would approximate the random errors that establish the relationship between the explanatory variables and the response variables. Therefore, if the residuals appear to behave randomly, it suggests that the model fits the data well. Figure 2 is the residual plots for the model. The plot shows evenly distributed random plots, which confirms that the model appears to fit the data set well. Thus, it can be concluded that the model adequately predicts compliance rate based on the distance between successive AWSC and signalized intersections.

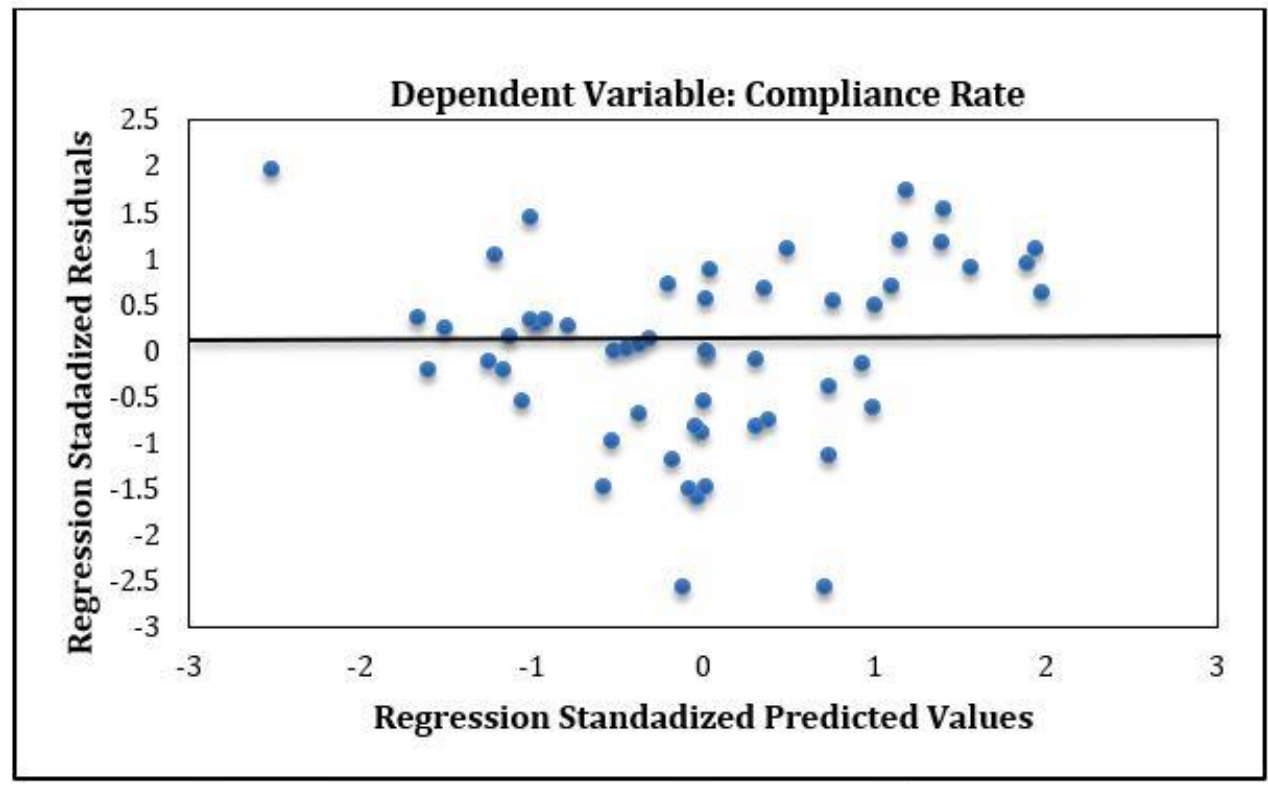

FIGURE 2 Residual plot for Compliance Rate

\section{Mean Absolute Percentage Error}

The Mean Absolute Percentage Error (MAPE) is a measure of quality of a model. MAPE indicates how much error is made in the predicted values as compared to the observed values. It is calculated by dividing the absolute errors of the predicted values by the observed values, and then averaging the obtained percentages. Thus,

$$
\begin{aligned}
& \mathrm{MAPE}=1 / \mathrm{n} \sum(|A-P| / A) \times 100 \\
& \text { where, } \\
& A=\text { Observed values } \\
& P=\text { Predicted values } \\
& n=\text { Number of observations }
\end{aligned}
$$

The MAPE analysis was performed using observed CR and the predicted CR values from the model. The analysis resulted in a MAPE of $6.1 \%$. This indicates that the model predicts CR with a $6.1 \%$ margin of error.

\section{DISCUSSION}

The study focused on improving compliance at AWCS by attempting to develop a model that could define the optimal distance of AWSC intersections from signalized intersections that could result in a relatively high rate of compliance. The study used 30segments that had AWSC intersections and signalized intersections upstream or downstream. The compliance observations were conducted via video playback for data compiled over a 6-months period. Other field data collected include traffic volumes entering the AWSC intersections and the distance between the upstream and/or downstream signalized intersections and the AWSC intersections.

In all 13,956 compliance observations were made. In addition, the minimum and maximum distances between the AWSC intersections and the signalized intersections were respectively 142 feet and 885 feet. The maximum compliance rate was determined to be approximately $92 \%$ and the minimum was $55 \%$. A model was then developed that predicts the compliance rate based on the distance from the upstream/downstream signalized intersection. The model had an $\mathrm{R}^{2}$ value of $74 \%$. The model also had a margin of error of $6.1 \%$.

Based on this study, it can be concluded that the closer the AWSC intersection is to the upstream/downstream signalized intersection, the lower the CR. From the model, it can be deduced that to attain a CR of $95 \%$ at an AWSC intersection, it should be approximately 1,300 feet away from the upstream or downstream signalized intersection. Drivers generally tend to "speed through" AWSC intersections if the signalized intersection on approach has a green interval, thereby violating the STOP sign.

\section{CONCLUSIONS}

The study revealed that there exists a strong relationship between the CR at AWSC intersections that are adjacent to signalized intersections and the distance between the two intersections. Lesser CR were reported at AWSC intersection with shorter distances to the next signalized 
intersection. In addition, the proposed model has a high explanatory power on the observed data. The model can therefore accurately predict the CR at AWSC intersections based on the distance between the AWSC and adjacent signalized intersections in Washington DC.

This model may not be applicable to other jurisdictions since driver behavior in Washington DC may differ from other urban jurisdictions. The study used 30 segments with different functional classifications and with distances of AWSC intersections less than 1,000 feet from next signalized intersections. Thus, future work may include validation of the model using field data, development of $\mathrm{CR}$ models for specific functional classification segments and including segments that have AWSC intersection distances of more than 1,000 feet from the next signalized intersections.

\section{ACKNOWLEDGEMENT}

The authors extend their appreciation to the U.S. Department of Transportation's University Transportation Centers Program and the staff of the Mineta Transportation Institute for funding and contributing to this study. Any opinions, findings, and conclusions or recommendations in this material are those of the authors and do not necessarily reflect the views of the U.S. Department of Transportation.

\section{REFERENCES}

[1] Center for Transportation Research and Education. Iowa State University (2007) - Access Management toolkit, Intersection Spacing and Traffic Signal Spacing. http://www.ctre.iastate.edu/research/access/toolkit/4.pdf. Accessed Sep. 9, 2017

[2] U.S Department of Transportation Federal Highway Administration. The National Intersection Safety Problem (2009). FHWA-SA-10-005

[3] National Highway and Transportation Safety Administration (2015)

https://crashstats.nhtsa.dot.gov/Api/Public/Publication/812384. Accessed Sep. 10, 2017

[4] Mounce, J.M. (1977). Driver Compliance with Stop-Sign Control at Low-Volume Intersections. Transportation Research Record 808, pp 30-37

[5] Woldeamanuel, M. (2012). Stopping Behavior of Drivers at Stop-Controlled Intersections: Compositional and Contextual Analysis. Journal of the Transportation Research Forum, Vol. 51, No. 3 (Fall 2012), pp. 109-123

[6] Mahadiow, A. (2017) STOP Sign Compliance at AWSCs in Close Proximity to Signalized Intersections. Transportation Research Board 96th Annual Meeting, Report/Paper Numbers: 17-06924

[7] Gordon, S., Robertson, D., Title, D. (198) A Study of Driver Noncompliance with Traffic Signals: Transportation Research Record 1168

[8] Elmitiny, Noor \& Yan, Xuedong \& Radwan, Essam \& Russo, Chris \& Elnashar, Dina. (2010). Classification analysis of driver's stop/go decision and red-light running violation. Accident; analysis and prevention. 42. 101-11. 10.1016/j.aap.2009.07.007.

[9] Fu, Chuanyun \& Pei, Yulong \& Wu, Yuqing \& Ha, Wa. (2013). The Influence of Contributory Factors on Driving Violations at Intersections: An Exploratory Analysis. Advances in Mechanical Engineering. 5. 905075-905075. 10.1155/2013/905075.

[10] Rice, E., Polanis, S.P. (2009). Stop Sign-Controlled Intersections. U.S. Department of Transportation Federal Highway Administration. FHWA-SA-09-010.

[11] Li, J., Qiu, T.Z. (2016) Improving Intersection Throughput Using Connected Vehicles. Resilient Infrastructure June 1-4, 2016 